\title{
Crisis deducing all physics laws, solution to the crisis and its applications to improved variational principle, improved Noether theorem and so on
}

\author{
C. Huang ${ }^{1,2 *}$ and Yong-Chang Huang ${ }^{3 \dagger}$ \\ ${ }^{1}$ Lawrence Berkeley National Laboratory, 1 Cyclotron Road, Berkeley CA 94720, USA \\ ${ }^{2}$ Department of Physics and Astronomy, Purdue University, \\ 525 Northwestern Avenue, W.Lafayette, IN 47907-2036, USA \\ ${ }^{3}$ Institute of Theoretical Physics, Beijing University of Technology, Beijing 100124, China
}

(Dated: August 14, 2020)

\begin{abstract}
This paper discovers that current variational principle and Noether theorem for both different physics systems and (in)finite freedom systems have missed the double extremum processes of the general extremum functional that is deduced by variational principle and necessarily taken in deducing all the physics laws, but these have not been corrected for over a century since Noether's proposing her theorem, which result in the crisis deducing all the physics laws. Using the double extremum processes of the general extremum functionals, the crisis and the hidden logic cycle problem in current variational principle and current Noether theorem are solved. Furthermore, the new mathematical and physical double extremum processes and their new mathematical and physical pictures for (in)finite freedom systems are discovered. The improved variational principle and improved Noether theorem are given, which are key useful to different branches of science.as key tools of studying and processing them.
\end{abstract}

Key words: fundamental interaction, variational principle, Noether theorem, physics law, unification theory

PACS numbers:

\section{I.Introduction}

A variational principle in science is to enables a problem being solved by using the calculus of variations, which optimizes the values of these quantities in the variational systems [1].

Fundamental physics laws can be expressed by a variational principle, which can give Euler-Lagrange equations and the corresponding convervation quantity $[2,3]$. Noether generalized the variational principle to a now called Noether theorem by finding the transformation symmetry properties of variational systems and giving both Euler-Lagrange equations and the many conservation quantities depending on the corresponding many symmetries $[4,5]$.

Current variational principle and Noether theorem have been extensively used in different branches of science and have become key tools of studying and processing the different branches, for examples:

In mathematics [1, 6-9]: (i) The extremum method for solving boundary-value problems; (ii) Variational principle in mathematical optimization; (iii) Variational principle in mathematical extremum problems; (iv) Variational principle in mathematical motion equations and invariant quantities; (v) The finite element method; ...... . In physics [2, 10-14]: (i) Fermat's principle in geometrical optics; (ii) Maupertuis' principle in classical mechanics; (iii) The principle of least action in mechanics, electromagnetic theory and so on; (iv) The variational method in quantum mechanics; (v) Gauss's principle of least con-

\footnotetext{
*Electronic address: c.huang0@hotmail.com

${ }^{\dagger}$ Electronic address: ychuang@bjut.edu.cn
}

straint and variational principle of least curvature; (vi) Hilbert's action principle in general relativity leading to Einstein field equations; (vii) Palatini variational principle; (viii) Variational principle in different field theories; ..... . In astronmy and astrophysics [15], in chemistry $[16,17]$, even in engineering [9] and so on.

Various variational principles and their applications are very well investigated, e.g., see [18-23]. Role of Noether's Theorem at the Deconfined Quantum Critical Point is studied [24], Noether's theorems and conserved currents in gauge theories in the presence of fixed fields are explored [25], furthermore, Noether's theorem and conserved quantities for the crystal- and ligand-field Hamiltonians invariant under continuous rotational symmetry are investigated [26].

In current variational principle and Noether theorem, there are the needs in advance to assume existing some conditions which are equivalent to Euler-Lagrange equations and conservation quantities, and then deducing Euler-Lagrange equations and conservation quantities, which are related to a hidden logic cycle problem and are not both exact and natural.

Furthermore, we find that all the investigations on variational principle and Noether theorem for different physics systems have missed the key studies on the double extremum processes related to the general extremum functional that is deduced via the least action principle and should be key largely taken in deducing al1 the physics laws, but the current variational principle and current Noether theorem have missed the general extreme functionals and their minimum extremums for over a century since Noether's proposing her theorem $[4,5]$, which result in the crisis of no objectively deducing all the physics laws. Using the studies on the double extremum 
processes related to the general extremum functionals in this paper, the crisis and the hidden logic cycle problem are solved, and the new physical pictures are discovered.

No losing generality, all physics laws always can be expressed as some equations, these equations always can be viewed as some Euler-Lagrange equations, the EulerLagrange equations always can be deduced by the general variational principle and/or Noether theorem [4, 5]. Therefore, there always is the crisis deducing all the physics laws. This paper wants to solve the crisis.

The arrangements of this paper are: Sect. 2 shows unification studies on variational principle and Noether theorem for finite freedom systems; Sect. 3 investigates crisis of deducing physics laws and its solution to the crisis for finite freedom systems; Sect. 4 gives unification studies on variational principle and Noether theorem for infinite freedom systems; Sect. 5 studies crisis of deducing physics laws and its solution to the crisis for infinite freedom systems; Sect. 6 shows discussions; Sect. 7 gives summary and conclusions.

II.Unification studies on variational principle and Noether theorem for finite freedom systems

The exact mathematical descriptions of the least action principle for a general case are: the variation of the integral ( i.e., the action ) of the Lagrangian $L$ during $\left[t_{1}, t_{2}\right]$ about $N$ generalized coordinates $q=\left(q_{1}, q_{2}, \ldots, q_{N}\right)$ is $[13,27]$

$\Delta A=A^{\prime}-A=\int_{t_{1}^{\prime}}^{t_{2}^{\prime}} L^{\prime}\left(q^{\prime}, \dot{q}^{\prime}, \ddot{q}^{\prime}, t^{\prime}\right) d t^{\prime}-\int_{t_{1}}^{t_{2}} L(q, \dot{q}, \ddot{q}, t) d t=0$.

It is no losing the generality, because the results of the systems with higher derivatives of $\mathrm{q}$ are the similar but more terms relevant to higher derivatives of $\mathrm{q}$.

Among them, the general infinitesimal transformations are [27-29]

$$
\begin{gathered}
t^{\prime}=t^{\prime}(q, \dot{q}, \ddot{q}, t, \alpha)=t+\Delta t=t+\varepsilon_{\sigma} \tau^{\sigma} \\
q_{i}^{\prime(r)}=q_{i}^{\prime(r)}(q, \dot{q}, \ddot{q}, t, \alpha)=q_{i}^{(r)}+\Delta q_{i}^{(r)}=q_{i}^{(r)}+\varepsilon_{\sigma}\left(\xi_{i}^{\sigma}\right)^{(r)}
\end{gathered}
$$

in which $r=0,1,2, \alpha=\left(\alpha_{1}, \alpha_{2}, \ldots, \alpha_{m}\right)$ are independent continuous variable parameters of Lie group $G$ and

$\left(\xi_{i}^{\sigma}\right)^{(r)}=\left.\frac{\partial q_{i}^{\prime}(r)(q, \dot{q}, \ddot{q}, t, \alpha)}{\partial \alpha_{\sigma}}\right|_{\alpha=0}, \sigma=1,2, \ldots, m ; r=0,1,2$,

$$
\tau^{\sigma}=\left.\frac{\partial t^{\prime}(q, \dot{q}, \ddot{q}, t, \alpha)}{\partial \alpha_{\sigma}}\right|_{\alpha=0}, \sigma=1,2, \ldots, m
$$

Eqs.(4) and (5) are the infinitesimal generating functions under the operation of group $\mathrm{G}, \varepsilon_{\sigma}(\sigma=1,2, \ldots, \mathrm{m})$ are independent infinitesimal parameters corresponding to $\alpha$, one dot and two dots denote the first and second order time derivatives respectively, the curve $q(t)$ is parameterized by time, and the path takes extremum corresponding $\triangle A=0$.

Doing as the well-known Refs. [2, 9, 13, 27, 29], we define

$L^{\prime}\left(q^{\prime}, \dot{q}^{\prime}, \ddot{q}^{\prime}, t^{\prime}\right)=L\left(q^{\prime}, \dot{q}^{\prime}, \ddot{q}^{\prime}, t^{\prime}\right)+\varepsilon_{\sigma} \frac{d \Omega^{\sigma}}{d t} \quad, \sigma=1,2, \ldots, m$.

Putting Eq.(6) into Eq.(1), one has

$\Delta A=\int_{t_{1}^{\prime}}^{t_{2}^{\prime}}\left[L\left(q^{\prime}, \dot{q}^{\prime}, \ddot{q}^{\prime}, t^{\prime}\right)+\varepsilon_{\sigma} \frac{d \Omega^{\sigma}}{d t}\right] d t^{\prime}-\int_{t_{1}}^{t_{2}} L(q, \dot{q}, \ddot{q}, t) d t=0$.

Using the technique of deducing Euler-Lagrange equations to simplify Eq.(7) and neglecting second-order infinitesimal quantities, we get

$$
\begin{gathered}
\Delta A=\int_{t_{1}}^{t_{2}}\left[\frac{d \Omega}{d t}+\sum_{i}\left[\frac{\partial L}{\partial q_{i}}-\frac{d}{d t} \frac{\partial L}{\partial \dot{q}_{i}}+\frac{d^{2}}{d t^{2}} \frac{\partial L}{\partial \ddot{q}_{i}}\right] \delta q_{i}+\right. \\
\left.\frac{d}{d t}\left[\sum_{i}\left(\frac{\partial L}{\partial \dot{q}_{i}} \delta q_{i}+\frac{\partial L}{\partial \ddot{q}_{i}} \delta \dot{q}_{i}-\frac{d}{d t} \frac{\partial L}{\partial \ddot{q}_{i}} \delta q_{i}\right)+L \Delta(t)\right]\right] d t
\end{gathered}
$$

where $\Omega=\varepsilon_{\sigma} \Omega^{\sigma}$. Eq.(8) is simplified as

$$
\Delta A=0=\int_{t_{1}}^{t_{2}}\left\{\sum_{i}\left[\frac{\partial L}{\partial q_{i}}-\frac{d}{d t} \frac{\partial L}{\partial \dot{q}_{i}}+\frac{d^{2}}{d t^{2}} \frac{\partial L}{\partial \ddot{q}_{i}}\right] \delta q_{i}+\right.
$$

$$
\left.\frac{d}{d t}\left[\sum_{i}\left(\frac{\partial L}{\partial \dot{q}_{i}} \delta q_{i}+\frac{\partial L}{\partial \ddot{q}_{i}} \delta \dot{q}_{i}-\frac{d}{d t} \frac{\partial L}{\partial \ddot{q}_{i}} \delta q_{i}\right)+L \Delta(t)+\Omega\right]\right\} d t .
$$

For Eq.(9), about the degree of freedom, there are still three different cases:

Case (i): When assuming

$$
\Delta A=\int_{t_{1}}^{t_{2}} \frac{d}{d t}\left[\sum_{i}\left(\frac{\partial L}{\partial \dot{q}_{i}} \delta q_{i}+\frac{\partial L}{\partial \ddot{q}_{i}} \delta \dot{q}_{i}-\frac{d}{d t} \frac{\partial L}{\partial \ddot{q}_{i}} \delta q_{i}\right)+L \Delta(t)+\Omega\right] d t
$$

using Eq.(10), one has

$$
\frac{\partial L}{\partial q_{i}}-\frac{d}{d t} \frac{\partial L}{\partial \dot{q}_{i}}+\frac{d^{2}}{d t^{2}} \frac{\partial L}{\partial \ddot{q}_{i}}=0,
$$

where $i=1,2, \ldots, N$, because $\delta q_{i}(\mathrm{i}=1,2, \ldots, \mathrm{N})$ are linear independent each other because $q_{i}$ are independent coordinates.

Using Eq.(10), we deduce conservation quantity

$$
\sum_{i}\left(\frac{\partial L}{\partial \dot{q}_{i}} \delta q_{i}+\frac{\partial L}{\partial \ddot{q}_{i}} \delta \dot{q}_{i}-\frac{d}{d t} \frac{\partial L}{\partial \ddot{q}_{i}} \delta q_{i}\right)+L \Delta(t)+\Omega=\text { const. }
$$


because

$$
\Delta q_{i}^{(r)}=\delta q_{i}^{(r)}(t)+q_{i}^{(r+1)}(t) \Delta t, r=0,1,2,
$$

Eq.(12) can be rewritten as

$$
\begin{aligned}
& \sum_{i}\left(\frac{\partial L}{\partial \dot{q}_{i}}\left(\Delta q_{i}-\dot{q}_{i} \Delta t\right)+\frac{\partial L}{\partial \ddot{q}_{i}}\left(\Delta \dot{q}_{i}-\ddot{q}_{i} \Delta t\right)\right. \\
& \left.-\frac{d}{d t} \frac{\partial L}{\partial \ddot{q}_{i}}\left(\Delta q_{i}-\dot{q}_{i} \Delta t\right)\right)+L \Delta(t)+\Omega=\text { const } .
\end{aligned}
$$

Eq.(14) is the result of variational principle.

Putting Eqs.(2) and (3) into Eq.(14), we deduce $\mathrm{m}$ conservation quantities of the systems

$$
\begin{aligned}
& \sum_{i}\left(\frac{\partial L}{\partial \dot{q}_{i}}\left(\xi_{i}^{\sigma}-\dot{q}_{i} \tau^{\sigma}\right)+\frac{\partial L}{\partial \ddot{q}_{i}}\left(\dot{\xi}_{i}^{\sigma}-\ddot{q}_{i} \tau^{\sigma}\right)\right. \\
& -\frac{d}{d t} \frac{\partial L}{\partial \ddot{q}_{i}}\left(\xi_{i}^{\sigma}-\dot{q}_{i} \tau^{\sigma}\right)+L \tau^{\sigma}+\Omega^{\sigma}=\text { const }^{\sigma} .
\end{aligned}
$$

where we have used that $\varepsilon_{\sigma}(\sigma=1,2, \ldots, m)$ are independent infinitesimal parameters. Namely, eq.(15) is the Noetther theorem's result.

We can see that both variational principle and Noether theorem all give the same Euler-Lagrange equations (11), but they give the convervation quantities are very different, i.e., Eq.(14) and Eq.(15) respectively.

Case (ii): When assuming that there exists Eq.(11), then putting Eq.(11) into Eq.(9), one has Eq.(10). In the following, there are the almost same discussions below Eq.(11) in Case (i).

III.Crisis of deducing physics laws and its solution to the crisis for finite freedom systems

Case (iii): Using Eq.(9) and the rule of merging like terms, we exactly have a general functional expression

$$
\begin{gathered}
\int_{t_{1}}^{t_{2}} \sum_{i}\left[\frac{\partial L}{\partial q_{i}}-\frac{d}{d t} \frac{\partial L}{\partial \dot{q}_{i}}+\frac{d^{2}}{d t^{2}} \frac{\partial L}{\partial \ddot{q}_{i}}\right] \delta q_{i} d t=-\int_{t_{1}}^{t_{2}} \frac{d}{d t}[ \\
\left.\sum_{i}\left(\frac{\partial L}{\partial \dot{q}_{i}} \delta q_{i}+\frac{\partial L}{\partial \ddot{q}_{i}} \delta \dot{q}_{i}-\frac{d}{d t} \frac{\partial L}{\partial \ddot{q}_{i}} \delta q_{i}\right)+L \Delta(t)+\Omega\right] d t .
\end{gathered}
$$

Eq.(16) comes from the general systems' taking extremum of the Lagrangian, but when the system has no Eq.(10) or Eq.(11), or no Eqs.(10) and (11), then the systems cannot give Euler-Lagrange equations and the corresponding conservation quantities. Namely, this case cannot give real physics laws, which is just the reason that current variational principle and current Noether theorem have missed the case (iii) [27-29].

Cases (i) and (ii) are necessary and sufficient conditions that just give real physics laws, and accordint to current variational principle and current Noether theorem [28, 29], case (iii) at all cannot give real physics laws.

Using Eq.(16) derived from the variational extremum, we can exactly define a general extremum functional

$$
\begin{gathered}
F=\int_{t_{1}}^{t_{2}} \sum_{i}\left[\frac{\partial L}{\partial q_{i}}-\frac{d}{d t} \frac{\partial L}{\partial \dot{q}_{i}}+\frac{d^{2}}{d t^{2}} \frac{\partial L}{\partial \ddot{q}_{i}}\right] \delta q_{i} d t=-\int_{t_{1}}^{t_{2}} \frac{d}{d t} \\
\left.\sum_{i}\left(\frac{\partial L}{\partial \dot{q}_{i}} \delta q_{i}+\frac{\partial L}{\partial \ddot{q}_{i}} \delta \dot{q}_{i}-\frac{d}{d t} \frac{\partial L}{\partial \ddot{q}_{i}} \delta q_{i}\right)+L \Delta(t)+\Omega\right] d t .
\end{gathered}
$$

The new general equal equation functional $F$ between the functional of deducing Euler-Lagrange equations having merged like terms and the functional of deducing the general conservation quantities having merged like terms is deduced by satisfying variational principle.

When the absolute value of the general extremum function $F$ is taken as zero, because the minimum absolute value of any function is zero, i.e., a general extremum ( because the general extremum function $F$ may generally take a lot of different values, e.g., arbitrary positive and/or negative values ), then we generally have

$$
\begin{gathered}
\int_{t_{1}}^{t_{2}} \sum_{i}\left[\frac{\partial L}{\partial q_{i}}-\frac{d}{d t} \frac{\partial L}{\partial \dot{q}_{i}}+\frac{d^{2}}{d t^{2}} \frac{\partial L}{\partial \ddot{q}_{i}}\right] \delta q_{i} d t=0 \\
=-\int_{t_{1}}^{t_{2}} \sum_{i} \frac{d}{d t}\left[\sum _ { i } \left(\frac{\partial L}{\partial \dot{q}_{i}} \delta q_{i}+\frac{\partial L}{\partial \ddot{q}_{i}} \delta \dot{q}_{i}\right.\right. \\
\left.\left.-\frac{d}{d t} \frac{\partial L}{\partial \ddot{q}_{i}} \delta q_{i}\right)+L \Delta(t)+\Omega\right] d t=0
\end{gathered}
$$

thus the first line of Eq.(18) is equivalent to case (ii), and the second and third lines of Eq.(18) are equivalent to case (i), these all can give physics laws. Namely, the general extremum functional $F$ takes the minimum absolute value, i.e., zero, all the physics laws can be deduced, otherwise, all the physics laws cannot be deduced. That is, Eq.(17) is deduced from the variational extremum, Eq.(18) is further taking the absolute extreme value zero, i.e., the minimum absolute extremum, of the general extremum functional $F$. Therefore, we, for the first time, discover that it is the double extreme values (i.e., the extreme functional $F$ 's extremum) that result in that all the physics laws can be deduced, otherwise, all the physics laws cannot be deduced.

Therefore, the systems first choose extreme value (i.e., via Eq.(1)) of the Lagrangian, and then we naturally deduce Eq.(9), there are needs as usual in advance to assume existing case (i) or (ii), because which are equivalent to Euler-Lagrange equations and conservation quantities, and then deducing Euler-Lagrange equations and conservation quantities, which are related to a hidden logic cycle problem and are not both exact and natural.

Actually, there naturally exists the general extremum functional $F$ so that we can choose the absolute extreme value zero of the general extremum functional $F$, then 
case (i) or (ii) can be naturally deduced ( e.g., see the studies below Eq.(18) ). Making these natural deductions reflects the systems' intrinsical properties, namely, the intrinsical mathematical and physical double extreme value procceses. Otherwise, the systems cannot get real physical laws. These results are supplementary developments of the current variational principle and current Noether theorem.

For all times, both the Lagrangian and the action contain the systems' dynamics, and the real appearance case is that the path taken by the systems during $\left[t_{1}, t_{2}\right]$ takes extreme value corresponding $\Delta A=0$, which means that the systems can not only choose but also make the least extremum choice and further choose the minimum absolute extremum of the general extremum functional $F$.

We discover that, up to now, all the investigations on variational principle and Noether theorem for different physics systems have missed the key studies on the double extremum processes related to the general extremum functional $F$ that is deduced via the least action principle and should be key largely taken in deducing all the physics laws, but the current variational principle and current Noether theorem have neglected the general extreme function $F$ and $F$ 's minimum extremum, which results in the crisis and the hidden logic cycle problem of no objectively deducing all physics laws. Using the studies on the double extremum processes related to the general extremum functional $F$ in this paper, the crisis and the hidden logic cycle problem are not only solved, but also the new mathematical and physical double extremum processes and their new mathematical and physical pictures are discovered. Therefore, the improved variantional principle and the improved Noether theorem for finite freedom systems are given, which solve the crisis and the hidden logic cycle problem.

IV.Unification studies on variational principle and Noether theorem for infinite freedom systems

For general field variables $X(x)=\{\Psi(x), \varphi(x)$, $\left.\omega_{\mu}(x), g_{\mu \nu}(x), \ldots,\right\}$, the exact mathematical description$\mathrm{s}$ of the least action principle for a general case are: the variation of the action about $N$ field components $X=\left(X^{1}, X^{2}, \ldots, X^{N}\right)$ is

$$
\begin{aligned}
\Delta A & =A^{\prime}-A=\int_{x_{1}^{\prime}}^{x_{2}^{\prime}} \mathcal{L}^{\prime}\left(X^{\prime}\left(x^{\prime}\right), \partial_{\alpha}^{\prime} X^{\prime}\left(x^{\prime}\right), \partial_{\alpha}^{\prime} \partial_{\beta}^{\prime} X^{\prime}\left(x^{\prime}\right), x^{\prime}\right) d x^{\prime 4} \\
& -\int_{x_{1}}^{x_{2}} \mathcal{L}\left(\left(X(x), \partial_{\alpha} X(x), \partial_{\alpha} \partial_{\beta} X(x), x\right) d x^{4}=0 .\right.
\end{aligned}
$$

in which the general infinitesimal transformations are $[28$, $29]$

$$
x^{\prime \mu}=x^{\mu}+\Delta x^{\mu}=x^{\mu}+\varepsilon_{\sigma}(x) \tau^{\mu \sigma}\left(x, X(x), \partial_{\alpha} X(x), \partial_{\alpha} \partial_{\beta} X(x)\right),
$$

$X^{\prime \alpha}\left(x^{\prime}\right)=X^{a}(x)+\varepsilon_{\sigma}(x) \xi^{a \sigma}\left(x, X(x), \partial_{\mu} X(x), \partial_{\mu} \partial_{\nu} X(x)\right)$ where $X^{\prime \alpha}\left(x^{\prime}\right)=X^{\alpha}(x)+\Delta X^{\alpha}(x), \omega=\left(\omega_{1}, \omega_{2}, \ldots, \omega_{m}\right)$ are independent continuous variable parameters of Lie group $G$ and

$$
\begin{aligned}
\tau^{\mu \sigma} & =\left.\frac{\partial x^{\mu}\left(x, X(x), \partial_{\mu} X(x), \partial_{\mu} \partial_{\nu} X(x), \omega\right)}{\partial \omega_{\sigma}}\right|_{\omega_{\sigma}=0}, \\
\xi^{a \sigma} & =\left.\frac{\partial X^{\alpha}\left(x, X(x), \partial_{\alpha} X(x), \partial_{\alpha} \partial_{\beta} X(x), \omega\right)}{\partial \omega_{\sigma}}\right|_{\omega_{\sigma}=0},
\end{aligned}
$$

where $\tau^{\mu \sigma}$ and $\xi^{a \sigma}(\sigma=1,2, \ldots, m)$ are infinitesimal transformation functions.

Eqs.(22) and (23) are the infinitesimal generating function under the operation of group $\mathrm{G}, \varepsilon_{\sigma}(\sigma=1,2, \ldots, \mathrm{m})$ are independent infinitesimal parameters corresponding to $\omega$.

Without loss of generality, we define

$\mathcal{L}^{\prime}\left(X^{\prime}\left(x^{\prime}\right), \partial_{\alpha}^{\prime} X^{\prime}\left(x^{\prime}\right), \partial_{\alpha} \partial_{\beta} X^{\prime}\left(x^{\prime}\right), x^{\prime}\right)=\mathcal{L}\left(X^{\prime}\left(x^{\prime}\right), \partial_{\alpha}^{\prime} X^{\prime}\left(x^{\prime}\right)\right.$, $\left.\partial_{\alpha}^{\prime} \partial_{\beta}^{\prime} X^{\prime}\left(x^{\prime}\right), x^{\prime}\right)+\varepsilon_{\sigma} \partial_{\mu} \Omega^{\sigma}\left(X(x), \partial_{\alpha} X(x), \partial_{\alpha} \partial_{\beta} X(x), x\right)$,

where $\sigma=1,2, \ldots, m$. Putting Eq.(24) into Eq.(19), one has

$$
\Delta A=\int_{x_{1}^{\prime}}^{x_{2}^{\prime}}\left[\mathcal{L}\left(X^{\prime}\left(x^{\prime}\right), \partial_{\alpha}^{\prime} X^{\prime}\left(x^{\prime}\right), \partial_{\alpha}^{\prime} \partial_{\beta}^{\prime} X^{\prime}\left(x^{\prime}\right), x^{\prime}\right)+\varepsilon_{\sigma} \partial_{\mu} \Omega^{\sigma}(X(\right.
$$

$\left.x), \partial_{\alpha} X(x), \partial_{\alpha} \partial_{\beta} X(x), x\right)-\mathcal{L}\left(X\left(x^{\prime}\right), \partial_{\alpha}^{\prime} X\left(x^{\prime}\right), \partial_{\alpha}^{\prime} \partial_{\beta}^{\prime} X\left(x^{\prime}\right), x^{\prime}\right)$

$\left.+\mathcal{L}\left(X\left(x^{\prime}\right), \partial_{\alpha}^{\prime} X\left(x^{\prime}\right), \partial_{\alpha}^{\prime} \partial_{\beta}^{\prime} X\left(x^{\prime}\right), x^{\prime}\right)\right] d x^{\prime 4}-\int_{x_{1}}^{x_{2}} \mathcal{L}(X(x)$,

$\left.\partial_{\alpha} X(x), \partial_{\alpha} \partial_{\beta} X(x), x\right) d x^{4}=\int_{x_{1}^{\prime}}^{x_{2}^{\prime}}\left[\delta\left(\mathcal{L}\left(X^{\prime}\left(x^{\prime}\right), \partial_{\alpha}^{\prime} X^{\prime}\left(x^{\prime}\right)\right.\right.\right.$,

$$
\begin{gathered}
\left.\partial_{\alpha}^{\prime} \partial_{\beta}^{\prime} X^{\prime}\left(x^{\prime}\right), x^{\prime}\right)+\varepsilon_{\sigma} \partial_{\mu} \Omega^{\sigma}\left(X(x), \partial_{\alpha} X(x), \partial_{\alpha} \partial_{\beta} X(x), x\right)+ \\
\left.\mathcal{L}+\frac{D \mathcal{L}}{D x^{\mu}} \Delta x^{\mu}\right] d x^{\prime 4}-\int_{x_{1}}^{x_{2}} \mathcal{L}\left(X(x), \partial_{\alpha} X(x), \partial_{\alpha} \partial_{\beta} X(x)\right. \\
x) d x^{4}=\int_{x_{1}}^{x_{2}}\left[\left(\frac{\partial \mathcal{L}}{\partial X^{a}} \delta X^{a}+\frac{\partial \mathcal{L}}{\partial X, a} \delta \partial_{\nu} X^{a}+\right.\right.
\end{gathered}
$$

$$
\left.\frac{\partial \mathcal{L}}{\partial X_{, \nu \rho}^{a}} \delta \partial_{\nu} \partial_{\rho} X^{a}\right) d x^{4}+\int_{x_{1}}^{x_{2}}\left[\varepsilon_{\sigma} \partial_{\mu} \Omega^{\sigma}+\mathcal{L}+\frac{D \mathcal{L}}{D x^{\mu}} \Delta x^{\mu}\right](
$$

$$
\left.1+\frac{\partial \Delta x^{\beta}}{\partial x^{\beta}}\right) d x^{4}-\int_{x_{1}}^{x_{2}} \mathcal{L}\left(X(x), \partial_{\alpha} X(x), \partial_{\alpha} \partial_{\beta} X(x), x\right) d x^{4}
$$


where $D \mathcal{L} / D x^{\mu}$ is whole derivative for the whole Lagrangian. Using the technique of deducing EulerLagrange equations to make Eq.(25) into order and neglecting two order infinitesimal quantities, we get

$$
\begin{aligned}
& \Delta A=\int_{M^{4}}\left\{\varepsilon_{\sigma} \partial_{\mu} \Omega^{\sigma}\left(X(x), \partial_{\alpha} X(x), \partial_{\alpha} \partial_{\beta} X(x), x\right)+\left[\frac{\partial \mathcal{L}}{\partial X^{a}}\right.\right. \\
& \left.-\partial_{\mu} \frac{\partial \mathcal{L}}{\partial X^{a}, \mu}+\partial_{\mu} \partial_{\nu} \frac{\partial \mathcal{L}}{\partial X^{a},{ }_{\mu \nu}}\right] \delta X^{a}+\partial_{\mu}\left[\left(\frac{\partial \mathcal{L}}{\partial X^{a},{ }_{\mu}}\right.\right. \\
& \left.\left.\left.-\partial_{\nu} \frac{\partial \mathcal{L}}{\partial X^{a},{ }_{\mu \nu}}\right) \delta X^{a}+\frac{\partial \mathcal{L}}{\partial X^{a},{ }_{\mu \nu}} \delta X^{a},{ }_{\nu}+\mathcal{L} \Delta x^{\mu}\right]\right\} d^{4} x
\end{aligned}
$$

Omitting the higher order infinitesimal quantities, Eq.(26) is simplified as

$$
\begin{aligned}
\Delta A= & 0=\int_{M^{4}}\left\{\left[\frac{\partial \mathcal{L}}{\partial X^{a}}-\partial_{\mu} \frac{\partial \mathcal{L}}{\partial X^{a}, \mu}+\partial_{\mu} \partial_{\nu} \frac{\partial \mathcal{L}}{\partial X^{a},{ }_{\mu \nu}}\right.\right. \\
& ] \delta X^{a}+\partial_{\mu}\left[\left(\frac{\partial \mathcal{L}}{\partial X^{a}, \mu}-\partial_{\nu} \frac{\partial \mathcal{L}}{\partial X^{a},{ }_{, \mu \nu}}\right) \delta X^{a}\right. \\
& \left.\left.+\frac{\partial \mathcal{L}}{\partial X^{a},{ }_{\mu \nu}} \delta X^{a},{ }_{\nu}+\mathcal{L} \Delta x^{\mu}+\Omega^{\mu}\right]\right\} d^{4} x
\end{aligned}
$$

in which $\Omega=\varepsilon_{\sigma} \Omega^{\sigma}(\sigma=1,2, \ldots, m)$ is one order infinitesimal quantity. Eq.(27) cannot directly give EulerLagrange equations, and there are some additional degrees of freedom.

For Eq.(27)), about the degree of freedom, there are still three cases:

Case (I): When assuming

$$
\begin{gathered}
\int_{M^{4}} \partial_{\mu}\left[\left(\frac{\partial \mathcal{L}}{\partial X^{a}, \mu}-\partial_{\nu} \frac{\partial \mathcal{L}}{\partial X^{a}, \mu \nu}\right) \delta X^{a}\right. \\
\left.\left.+\frac{\partial \mathcal{L}}{\partial X^{a}{ }_{, \mu \nu}} \delta X^{a}{ }_{, \nu}+\mathcal{L} \Delta x^{\mu}+\Omega^{\mu}\right]\right\} d^{4} x=0,
\end{gathered}
$$

using Eq.(28), one has

$$
\frac{\partial \mathcal{L}}{\partial X^{a}}-\partial_{\mu} \frac{\partial \mathcal{L}}{\partial X^{a}, \mu}+\partial_{\mu} \partial_{\nu} \frac{\partial \mathcal{L}}{\partial X^{a}, \mu \nu}=0
$$

because $\delta X^{a}(a=1,2, \ldots, N)$ are independent each other. Eq.(29) are just the usual Euler-Lagrange equations.

Using Eq.(28), we deduce a general continuous equation

$$
\begin{aligned}
& \partial_{\mu} J^{\mu}=0=\partial_{\mu}\left[\left(\frac{\partial \mathcal{L}}{\partial X^{a},{ }_{\mu}}-\partial_{\nu} \frac{\partial \mathcal{L}}{\partial X^{a},{ }_{\mu \nu}}\right) \delta X^{a}+\right. \\
& \left.\frac{\partial \mathcal{L}}{\partial X^{a},{ }_{\mu \nu}} \delta X^{a}{ }_{, \nu}+\mathcal{L} \Delta x^{\mu}+\Omega^{\mu}\right]
\end{aligned}
$$

and its general conservation current

$$
J^{\mu}=\left(\frac{\partial \mathcal{L}}{\partial X^{a}, \mu}-\partial_{\nu} \frac{\partial \mathcal{L}}{\partial X^{a},{ }_{\mu \nu}}\right) \delta X^{a}+\frac{\partial \mathcal{L}}{\partial X^{a},{ }_{\mu \nu}} \delta X^{a}{ }_{, \nu}+\mathcal{L} \Delta x^{\mu}+\Omega^{\mu}
$$

Because

$$
\delta X^{a}=\Delta X^{a}-X^{a},{ }_{\nu} \Delta x^{\nu} ; \delta X,{ }_{\beta}^{a}=\Delta X,{ }_{\beta}^{a}-X,{ }_{\beta \nu}^{a} \Delta x^{\nu},
$$

Eqs. (30) and (31) can be rewritten as

$$
\begin{array}{r}
\partial_{\mu} J^{\mu}=\partial_{\mu}\left[\left(\frac{\partial \mathcal{L}}{\partial X^{a}{ }_{, \mu}}-\partial_{\nu} \frac{\partial \mathcal{L}}{\partial X^{a},{ }_{\mu \nu}}\right)\left(\Delta X^{a}-X^{a},{ }_{\nu^{\prime}} \Delta x^{\nu^{\prime}}\right)+\right. \\
\left.\frac{\partial \mathcal{L}}{\partial X^{a}{ }_{, \mu \nu}}\left(\Delta X_{,{ }_{\nu}}{ }^{a}-X^{a}{ }_{, \nu \nu^{\prime}} \Delta x^{\nu^{\prime}}\right)+\mathcal{L} \Delta x^{\mu}+\Omega^{\mu}\right]=0
\end{array}
$$

and a general conservation current

$$
\begin{gathered}
J^{\mu}=\left(\frac{\partial \mathcal{L}}{\partial X^{a}, \mu}-\partial_{\nu} \frac{\partial \mathcal{L}}{\partial X^{a},{ }_{\mu \nu}}\right)\left(\Delta X^{a}-X^{a},{ }_{\nu^{\prime}} \Delta x^{\nu^{\prime}}\right)+ \\
\frac{\partial \mathcal{L}}{\partial X^{a}{ }_{, \mu \nu}}\left(\Delta X_{,{ }_{\nu}}^{a}-X^{a}{ }_{, \nu \nu^{\prime}} \Delta x^{\nu^{\prime}}\right)+\mathcal{L} \Delta x^{\mu}+\Omega^{\mu}
\end{gathered}
$$

Namely, eq.(34) is the variational principle's result.

Using Eqs.(20) and (21), we achieve m continuos equations and their conservative currents

$$
\begin{aligned}
& \partial_{\mu} J^{\mu \sigma}=\partial_{\mu}\left[\left(\frac{\partial \mathcal{L}}{\partial X^{a}, \mu}-\partial_{\nu} \frac{\partial \mathcal{L}}{\partial X^{a}{ }_{, \mu \nu}}\right)\left(\xi^{a \sigma}-X^{a}, \nu^{\prime} \tau^{\nu^{\prime} \sigma}\right)+\right. \\
& \left.\frac{\partial \mathcal{L}}{\partial X^{a},{ }_{\mu \nu}}\left(\xi_{, \nu}^{a \sigma}-X^{a}{ }_{, \nu \nu^{\prime}} \tau^{\nu^{\prime} \sigma}\right)+\mathcal{L} \tau^{\mu \sigma}+\Omega^{\mu \sigma}\right]=0 \\
& \begin{array}{c}
J^{\mu \sigma}=\left(\frac{\partial \mathcal{L}}{\partial X^{a}{ }_{, \mu}}-\partial_{\nu} \frac{\partial \mathcal{L}}{\partial X^{a},{ }_{\mu \nu}}\right)\left(\xi^{a \sigma}-X^{a},{ }_{\nu^{\prime}} \tau^{\nu^{\prime} \sigma}\right)+ \\
\frac{\partial \mathcal{L}}{\partial X^{a}{ }_{, \mu \nu}}\left(\xi_{,{ }_{\nu}}^{a \sigma}-X^{a},{ }_{\nu \nu^{\prime}} \tau^{\nu^{\prime} \sigma}\right)+\mathcal{L} \tau^{\mu \sigma}+\Omega^{\mu \sigma}
\end{array}
\end{aligned}
$$

where we have used that $\varepsilon_{\sigma}(\sigma=1,2, \ldots, m)$ are independent infinitesimal parameters. Namely, eq.(36) is the Noetther theorem's result.

Using Eqs.(34) and (36) and $\int_{M^{3}} \partial_{0} J^{0} d V=$ $-\int_{M^{2}} J^{i} d S_{i} \rightarrow 0,\left(S_{i} \rightarrow \infty, J^{i} \rightarrow 0\right)$, we achieve conservation charges of variational principle and Noether theorem, respectively

$$
\begin{gathered}
Q_{v p}=\int_{M^{3}}\left[\left(\frac{\partial \mathcal{L}}{\partial X^{a}, 0}-\partial_{\nu} \frac{\partial \mathcal{L}}{\partial X^{a}, 0 \nu}\right)\left(\Delta X^{a}-X^{a},{ }_{\nu^{\prime}} \Delta x^{\nu^{\prime}}\right)+\right. \\
\left.\frac{\partial \mathcal{L}}{\partial X^{a}, 0 \nu}\left(\Delta X_{, \nu}^{a}-X^{a},{ }_{\nu \nu^{\prime}} \Delta x^{\nu^{\prime}}\right)+\mathcal{L} \Delta x^{0}+\Omega^{0}\right] d V,
\end{gathered}
$$




$$
\begin{aligned}
Q_{N t}^{\sigma}= & \int_{M^{3}}\left[\left(\frac{\partial \mathcal{L}}{\partial X^{a}, 0}-\partial_{\nu} \frac{\partial \mathcal{L}}{\partial X^{a}, 0 \nu}\right)\left(\xi^{a \sigma}-X^{a},{ }_{\nu^{\prime}} \tau^{\nu^{\prime} \sigma}\right)+\right. \\
& \frac{\partial \mathcal{L}}{\partial X^{a}, 0 \nu}\left(\xi_{, \nu}^{a \sigma}-X^{a}{ }_{, \nu \nu^{\prime}} \tau^{\nu^{\prime} \sigma}\right)+\mathcal{L} \tau^{0 \sigma}+\Omega^{0 \sigma} d V(38)
\end{aligned}
$$

where $\sigma=1,2, \ldots, m$. We can see that both variational principle and Noether theorem all give the same EulerLagrange equations (29), but they give the convervation currents (charges) are very different, i.e., Eq.(34) and Eq.(36) (Eq.(37) and Eq.(38)) respectively.

Case (II): When assuming that there are Eq.(29), then putting Eq.(29) into Eq.(27), one has Eq.(28). In the following, there the almost same discussions below Eq.(29) in Case (I).

V.Crisis of deducing physics laws and its solution to the crisis for infinite freedom systems

Case (III): Using Eq.(27), we generally have

$$
\int_{M^{4}}\left\{\left[\frac{\partial \mathcal{L}}{\partial X^{a}}-\partial_{\mu} \frac{\partial \mathcal{L}}{\partial X^{a}, \mu}+\partial_{\mu} \partial_{\nu} \frac{\partial \mathcal{L}}{\partial X^{a}, \mu \nu}\right] \delta X^{a} d^{4} x=-\int_{M^{4}} \partial_{\mu}[(\right.
$$$$
\left.\left.\left.\frac{\partial \mathcal{L}}{\partial X^{a},_{\mu}}-\partial_{\nu} \frac{\partial \mathcal{L}}{\partial X^{a},{ }_{\mu \nu}}\right) \delta X^{a}+\frac{\partial \mathcal{L}}{\partial X^{a},{ }_{\mu \nu}} \delta X^{a},{ }_{\nu}+\mathcal{L} \Delta x^{\mu}+\Omega^{\mu}\right]\right\} d^{4} x
$$

Eq.(39) comes from the general systems' taking extremum of the Lagrangian, but when the systems have no Eq.(28) or Eq.(29), or no Eqs.(28) and (29), then the systems cannot give Euler-Lagrange equations and the corresponding conservation quantities. Namely, this case cannot give real physics laws, which is just the reason that current variational principle and current Noether theorem have missed the case (III) [28, 29].

Cases (I) and (II) are necessary and sufficient conditions that just give real physics laws, and accordint to current variational principle and current Noether theorem [28, 29], case (III) at all cannot give real physics laws.

Using Eq.(39) derived from the variational extremum, we can exactly define a general extremum functional

$$
\begin{aligned}
G= & \int_{M^{4}}\left[\frac{\partial \mathcal{L}}{\partial X^{a}}-\partial_{\mu} \frac{\partial \mathcal{L}}{\partial X^{a},_{\mu}}+\partial_{\mu} \partial_{\nu} \frac{\partial \mathcal{L}}{\partial X^{a}, \mu \nu}\right] \delta X^{a} d^{4} x \\
=- & -\int_{M^{4}} \partial_{\mu}\left[\left(\frac{\partial \mathcal{L}}{\partial X^{a},{ }_{\mu}}-\partial_{\nu} \frac{\partial \mathcal{L}}{\partial X^{a},{ }_{\mu \nu}}\right) \delta X^{a}\right. \\
& \left.+\frac{\partial \mathcal{L}}{\partial X^{a}{ }_{, \mu \nu}} \delta X^{a},_{\nu}+\mathcal{L} \Delta x^{\mu}+\Omega^{\mu}\right] d^{4} x
\end{aligned}
$$

The new general equal equation functional $G$ between the functional of deducing Euler-Lagrange equations having merged like terms and the functional of deducing the general conservation quantities having merged like terms is deduced by satisfying variational principle.

When the absolute value of the general extremum function $G$ is taken as zero, because the minimum absolute value of any function is zero, i.e., a general extremum ( because the general extremum functional $G$ may generally take a lot of different values, e.g., arbitrary positive and/or negative values ), then we generally have

$$
\begin{gathered}
\int_{M^{4}}\left[\frac{\partial \mathcal{L}}{\partial X^{a}}-\partial_{\mu} \frac{\partial \mathcal{L}}{\partial X^{a},{ }_{\mu}}+\partial_{\mu} \partial_{\nu} \frac{\partial \mathcal{L}}{\partial X^{a}, \mu \nu}\right] \delta X^{a} d^{4} x=0 \\
=-\int_{M^{4}} \partial_{\mu}\left[\left(\frac{\partial \mathcal{L}}{\partial X^{a},{ }_{\mu}}-\partial_{\nu} \frac{\partial \mathcal{L}}{\partial X^{a},{ }_{\mu \nu}}\right) \delta X^{a}\right. \\
\left.+\frac{\partial \mathcal{L}}{\partial X^{a},{ }_{\mu \nu}} \delta X^{a},{ }_{\nu}+\mathcal{L} \Delta x^{\mu}+\Omega^{\mu}\right] d^{4} x=0
\end{gathered}
$$

thus the first line of Eq.(41) is equivalent to case (II), and the sum of the second and third lines of Eq.(41) are equivalent to case (I), these all can give physics laws. Namely, the general extremum function $G$ takes the minimum absolute value, i.e., zero, all the physics laws can be deduced. Otherwise, all the physics laws cannot be deduced. That is, Eq.(39) is deduced from the variational extremum, Eq.(41) is further taking the absolute extreme value zero, i.e., the minimum absolute extremum, of the general extremum functional $G$, therefore, we, for the first time, discover that it is the double extreme values (i.e., the extreme functional $G$ 's extremum) that result in that all the physics laws can be deduced, otherwise, all the physics laws cannot be deduced.

Therefore, the systems first choose extreme value (i.e., via Eq.(19)) of the Lagrangian, and then we naturally deduce Eq.(27), there are needs as usual in advance to assume existing case (I) or (II), because which are equivalent to Euler-Lagrange equations and conservation quantities, and then deducing Euler-Lagrange equations and conservation quantities, which are related to a hidden logic cycle problem and are not both exact and natural.

Actually, there naturally exists the general extremum functional $G$ so that we can choose the absolute extreme value zero of the general extremum functional $G$, then case (I) or (II) can be naturally deduced ( e.g., see the studies below Eq.(41) ). Making these natural deductions reflects the systems' intrinsical properties, namely, the intrinsical mathematical and physical double extreme value procceses. Otherwise, the systems cannot get real physical laws. These results are supplementary developments of the current variational principle and current Noether theorem for infinite freedom systems.

We discover that, up to now, all the investigations on variational principle and Noether theorem for different physics systems and infinite freedom systems have missed the key studies on the double extremum processes related to the general extremum functional $G$ that is deduced via the least action principle and should be key largely taken in deducing all the physics laws, but the current variational principle and current Noether theorem for infinite freedom systems have missed the general extreme functional $G$ and $G$ 's minimum extremum, which result$\mathrm{s}$ in the crisis and the hidden logic cycle problem of no objectively deducing all physics laws. Using the studies 
on the double extremum processes related to the general extremum functional $G$ in this paper, the crisis and the hidden logic cycle problem are not only solved, but also the new mathematical and physical double extremum processes and their new mathematical and physical pictures are discovered. Therefore, the improved variantional principle and the improved Noether theorem for infinite freedom systems are given, which solve the crisis and the hidden logic cycle problem.

\section{Discussions}

Using Eq.(17) derived from the variational extremum, we have a general extremum functional expression for finite freedom systems

$$
\begin{gathered}
f=\sum_{i}\left[\frac{\partial L}{\partial q_{i}}-\frac{d}{d t} \frac{\partial L}{\partial \dot{q}_{i}}+\frac{d^{2}}{d t^{2}} \frac{\partial L}{\partial \ddot{q}_{i}}\right] \delta q_{i}= \\
-\frac{d}{d t}\left[\sum_{i}\left(\frac{\partial L}{\partial \dot{q}_{i}} \delta q_{i}+\frac{\partial L}{\partial \ddot{q}_{i}} \delta \dot{q}_{i}-\frac{d}{d t} \frac{\partial L}{\partial \ddot{q}_{i}} \delta q_{i}\right)+L \Delta(t)+\Omega\right] .
\end{gathered}
$$

where $f$ can take any functional value and $F=\int_{t_{1}}^{t_{2}} f d t$.

When the absolute value of the general extremum functional $f$ is taken as zero, namely, taking the minimum absolute extreme value of the general extremum functional $f$, i.e., the general extremum functional $f$ 's extremum, that is, the double extremum process, Eq.(42) can directly deduce Euler-Lagrange equations due to the linear independent properties of $\delta q_{i}$ and the general conservation quantity due to having taken the second line of Eq.(42) as zero.

Using Eq.(40) derived from the variational extremum, we deduce a general extremum functional expression for infinite freedom systems

$$
\begin{gathered}
g=\left[\frac{\partial \mathcal{L}}{\partial X^{a}}-\partial_{\mu} \frac{\partial \mathcal{L}}{\partial X^{a}, \mu}+\partial_{\mu} \partial_{\nu} \frac{\partial \mathcal{L}}{\partial X^{a},{ }_{\mu \nu}}\right] \delta X^{a}= \\
-\partial_{\mu}\left[\left(\frac{\partial \mathcal{L}}{\partial X^{a}, \mu}-\partial_{\nu} \frac{\partial \mathcal{L}}{\partial X^{a},{ }_{, \mu \nu}}\right) \delta X^{a}+\frac{\partial \mathcal{L}}{\partial X^{a},{ }_{\mu \nu}} \delta X^{a}{ }_{,}+\mathcal{L} \Delta x^{\mu}+\Omega^{\mu}\right]
\end{gathered}
$$

where $g$ can take any functional value and $G=\int_{M^{4}} g d^{4} x$.

When the absolute value of the general extremum functional $g$ is taken as zero, namely, taking the minimum absolute extreme value of the general extremum functional $g$, i.e., the general extremum functional $g$ 's extremum, that is, the double extremum process, Eq.(43) can directly deduce Euler-Lagrange equations due to the linear independent properties of $\delta X^{a}$ and the general conservation current due to having taken the second line of Eq.(43) as zero.

All the investigations on functionals $F$ and $G$ in this paper give the corresponding integral descriptions, using functional $f$ and $g$ we can give the corresponding differantial descriptions, the two descriptions are entirely equivalent, thus we don't repeat more here.

\section{Summary and conclutions}

For the systems with Lagrangian and symmetry for finite (infinite) freedom systems, people can deduce Euler-Lagrange equations and corresponding conservation quantities by utilizing the variational principle and Noether theorem. But we discover the fact that the systems generally have extra intrinsical freedoms of choice. And if not assuming to exist Eq.(10) or (11) (Eq.(28) or (29)), then the Lagrange systems cannot give true physical laws. Actually, Eqs.(10) and (11) (Eqs.(28) and (29)) are equivalent to Euler-Lagrange equations and conservation quantities, and then deducing Euler-Lagrange equations and conservation quantities, which are related to a hidden logic cycle problem and are not both exact and natural.

The systems first choose extreme value, and then must choose the minimum absolute extremum of the general extremum functional $F(G)$, then cases (i) or (ii) (cases (I) or (II)) can be naturally deduced. Making these deductions show the systems' intrinsical properties of taking double extreme values, otherwise cannot get real physical laws according exact deduction logic. These results are supplementary developments of the curren$\mathrm{t}$ variational principle and current Noether theorem for finite (infinite) freedom system.

This paper discovers, up to now, all the studies on variational principle and Noether theorem for both different physics systems and finite (infinite) freedom systems have neglected the key studies on the double extremum processes of the general extremum functional $F(G)$ that is deduced by the least action principle and must be key largely taken in deducing all the physics laws, but these have not been done, which result in the crisis of deducing all physics laws. Using the above studies on the double extremum processes of the general extremum functional $F(G)$ in this paper, i.e., on the double extreme values, the crisis and the hidden logic cycle problem are not only solved, but also the new mathematical and physical double extremum processes and their new mathematical and physical pictures are discovered. Therefore, the improved variantional principle and the improved Noether theorem for (in)finite freedom systems are given in this paper, which solve the crisis and the hidden logic cycle problem.

Therefore, this paper opens a new area of research on variational principle and Noether theorem for finite (infinite) freedom systems by choosing optima of the double extreme values to explain origins of physics laws etc., and will significantly influence the research of others in different branches of science, because the least action principle or variational principle and Noether theorem are the key firm bases in modern physics, and we just discover new right avenues of research within the established variational principle and Noether theorem for finite (infinite) freedom systems, their applications and so on in modern sciences, and all the relevant current articles and (text)books should be supplied and updated.

Acknowledgments: The work is supported by NSF 
through grants PHY-0805948, DOE through grant DEFG02- 91ER40681, National Natural Science Foundation of China (No. 11875081).

References
[1] Morris Kline, Mathematical Thought from Ancient to Modern Times. New York: Oxford University Press (1972).

[2] Philippe Blanchard, G. M. Hayes, Variational Methods in Mathematical Physics: A Unified Approach (Theoretical and Mathematical Physics), Springer (2011).

[3] Richard Feynman, The Character of Physical Law, Modern Library, (1965).

[4] Noether, E., Invariante Variationsprobleme, Nachrichten von der Gesellschaft der Wissenschaften zu Gottingen, Mathematisch-Physikalische Klasse. 1918: 235-257.

[5] EDITORIAL, Celebrate the mathematics of Emmy Noether, Nature 561, 149-150 (2018).

[6] Ekeland, Ivar (1979). "Nonconvex minimization problems". Bulletin of the American Mathematical Society. New Series. 1 (3): 443-474.

[7] Yong-Chang Huang, Unified Expressions of All Differential Variational Principles, Mechanics Research Communications, 30, 6 (2003) 567, and its references.

[8] Yong-Chang Huang, X. G. Lee and M. X. Shao, Unified Expressions Of All Integral Variational Principles, Modern Physics Letters, A21(2006) 1107.

[9] Kevin W. Cassel, Variational Methods with Applications in Science and Engineering, Cambridge University Press, 2013.

[10] Cornelius Lanczos, The Variational Principles of Mechanics (4th ed.). Dover. University of Toronto Press (1974).

[11] Adhikari S. K., Variational Principles for the Numerical Solution of Scattering Problems, New York: Wiley, 1998.

[12] Yong-Chang Huang, L. Liao and X. G. Lee, FaddeevJackiw canonical path integral quantization for a general scenario, its proper vertices and generating functionals, The European Physical Journal, C60 (2009) 481-487.

[13] Hand L. N., Finch J. D., Analytical Mechanics, England: Cambridge University Press, 2008.

[14] M. E. Peskin and D. V. Schroeder, An Introduction to Quantum Field Theory (World Publishing Corp., 2006).

[15] John Venables, "The Variational Principle and some applications". Dept of Physics and Astronomy, Arizona State University, Tempe, Arizona (Graduate Course:
Quantum Physics)

[16] Nesbet R. K., Variational Principles and Methods In Theoretical Physics and Chemistry, New York: Cambridge U.P., 2003.

[17] Epstein S. T., The Variation Method in Quantum Chemistry, New York: Academic, 1974.

[18] Marco Piccardo, Paul Chevalier, Benedikt Schwarz et al, Frequency-Modulated Combs Obey a Variational Principle, Phys. Rev. Lett. 122, 253901 (2019).

[19] Alexander G. Abanov and Gustavo M. Monteiro, FreeSurface Variational Principle for an Incompressible Fluid with Odd Viscosity, Phys. Rev. Lett. 122, 154501 (2019).

[20] Pablo M. Piaggi and Michele Parrinello, MultithermalMultibaric Molecular Simulations from a Variational Principle, Phys. Rev. Lett. 122, 050601 (2019).

[21] Dallas R. Trinkle, Variational Principle for Mass Transport, Phys. Rev. Lett. 121, 235901 (2018).

[22] Hendrik Weimer, Variational Principle for Steady States of Dissipative Quantum Many-Body Systems, Phys. Rev. Lett. 114, 040402 (2015).

[23] Jutho Haegeman, J. Ignacio Cirac, Tobias J. Osborne et al, Time-Dependent Variational Principle for Quantum Lattices, Phys. Rev. Lett. 107, 070601 (2011).

[24] Nvsen Ma, Yi-Zhuang You, and Zi Yang Meng, Role of Noether's Theorem at the Deconfined Quantum Critical Point, Phys. Rev. Lett. 122, 175701 (2019).

[25] Gáor Zsolt Tóh, Noether's theorems and conserved currents in gauge theories in the presence of fixed fields, Phys. Rev. D 96, 025018 (2017).

[26] C. Rudowicz and J. Qin, Noether's theorem and conserved quantities for the crystal- and ligand-field Hamiltonians invariant under continuous rotational symmetry, Phys. Rev. B 67, 174420 (2003).

[27] Kibble T. W. B., Classical Mechanics, European Physics Series, McGraw-Hill (UK), 1973, ISBN 0-07-084018-0.

[28] Gitman D. M., Tyutin I. V., Quantization of Fields with Constraints, Springer-Verlag, Berlin, 1990.

[29] Li Ziping and Jiang Jinhuan, Symmetries in Constrained Canonical Systems, Science Press, New York, 2002. 